\title{
The Co-symmedian System of Tetrahedra inscribed in a Sphere.
}

\author{
By Dr W. L. MARR. \\ (Read and Received 19th June 1919.)
}

This paper deals with a system of tetrahedra in a sphere corresponding to the co-symmedian system of triangles in a circle. Such a system of tetrahedra, so far as the writer knows, has not been hitherto discussed. The condition that a tetrahedron may have a symmedian point is given in Wolstenholme's "Problems" $(1878)$.

1. When has a tetrahedron a symmedian point, i.e. when are the lines joining the vertices to the poles of the opposite faces with respect to the circumsphere concurrent?

Let $A B C D$ be a tetrahedron, $a$ the pole of $B C D, c$ the pole of $A B D$. Suppose that $C c$ and $A a$ meet in $K$.

The points $a$ and $c$ lie on the tangent planes at $B$ and $D$, and therefore $a c$ is the intersetion of these tangent planes. But ac and $A C$ are coplanar, therefore the tangent planes at $B$ and $D$ meet on $A C$.

But the tangent plane at $D$ meets $A C$ where the tangent at $D$ with respect to the circle $A C D$ meets $A C$ at $X$ (say), and the tangent plane at $B$ meets $A C$ where the tangent at $B$ with respect to the circle $A B C$ meets $A C$ also at $X$.

$$
\frac{X A}{X C}=\frac{A D^{2}}{D C^{2}}=\frac{A B^{2}}{B C^{2}}
$$

therefore

$$
A D . B C=A B . D C \text {. }
$$

Hence, that there may be a symmedian point, the tetrahedron must be equianharmonic ( $v$. Harkness and Morley).

2. The symmedian point of an equianharmonic tetrahedron is the point of intersection of the lines joining the vertices to the symmedian points of the opposite faces.

The line from $A$ to the pole of $B D$ (circle $A B D$ ) is a symmedian line of $A B D$. But the pole of $B D$ lies on $c a$, therefore the 
symmedian line of $A B D$ drawn from $A$ lies in the plane of $A c a$, i.e. $A C K$. The symmedian line of $A B D$ from $B$ lies in the plane of $B C K$.

Therefore, if $k_{3}$ be the symmedian point of $A B D$, it will lie on the intersection of $A C K$ and $B C K$, i.e. $C, K, k_{3}$ are collinear.

Further, since $k_{1}, k_{n}$ etc., lie within their triangles, $K$ will lie within the tetrabedron.

3. An equianharmonic tetrahedron has two Isodynamic points.

Let $H$ be an isodynamic point, then from the foregoing the following relations are possible :-

$$
\begin{aligned}
& H A \cdot B C=H B \cdot C A=H C \cdot A B \\
& H A \cdot D C=\text { etc. } ; H B \cdot A D=\text { etc. } ; H B \cdot C D=\text { etc., }
\end{aligned}
$$

i.e. $H$ is the vertex of four harmonic tetrahedra, the opposite faces being the faces of the original tetrahedron. $H$ lies on the circle which is the intersection of the three Apollonian spheres of $B D C$ and on the circle which is the intersection of the three Apollonian spheres of $A D C$. These two circles lie on the Apollonian sphere of $D C$ and cut in two real-as will be proved presently-points, $H_{1}$ and $H_{2}$.

These points are common to the six Apollonian spheres.

4. The circumcentre is collinear with the two isodynamic points.

The tangents from $O$, the circumcentre, to the Apollonian spheres are all equal to the radius of the circumsphere, and since these six spheres cut in $H_{1}$ and $H_{2}$, the three points $O, H_{1}, H_{2}$ must be collinear.

5. The isodynamic points, the circumcentre, and the symmedian point are collinear and form a harmonic ratio.

Consider the Apollonian sphere through $A$ and $C$. The centre of this sphere will lie on $B D$, viz., $L$. Since $A\left(L D k_{3} B\right)$ is harmonic, $k_{3}$ lies on the polar plane of $L$ with respect to the circumsphere. But $L A$ and $L C$ are tangents to the sphere, and therefore $A C$ lies on the polar plane.

Hence $A C k_{3}$, i.e. $A C K$, is the polar plane of $L$ with respect to the circumsphere. Since the circum- and Apollonian spheres cut orthogonally, $A C K$ is the plane of intersection of the two spheres. 
Let $A K$ meet the circumsphere again in $A^{\prime}$, etc., then

$$
A K \cdot A^{\prime} K=B K \cdot B^{\prime} K=\text { etc. }
$$

But $A, A^{\prime}$, etc., are points on the Apollonian spheres, hence $K$ must lie on the line of intersection.

Since $A C K$ is the polar plane of $O$ with respect to the Apollonian sphere whose centre is $L, O H_{1} K H_{2}$ is harmonic. As $K$ is within the circumsphere, it must also lie within the Apollonian sphere, and hence $H_{1}$ and $H_{2}$ are real points.

6. Since the points $A^{\prime} B^{\prime} C^{\prime} D^{\prime}$, as above defined, have the same circum- and Apollonian spheres, $A^{\prime} B^{\prime} C^{\prime} D^{\prime}$ will have the same symmedian points.

7. The faces of $A B C D$ and $A^{\prime} B^{\prime} C^{\prime} D^{\prime}$ touch an ellipsoid whose section perpendicular to $O K$ is circular.

If

$$
B C=a, \quad C A=b, \quad A B=c,
$$

and

$$
D A=\frac{k}{a}, \quad D B=\frac{k}{b}, \quad D C=\frac{k}{c},
$$

and if $m=\frac{a b c}{k}$ it can be readily shewn that the ellipsoid is :-

$$
\frac{x^{2}}{a^{4}}+\frac{y^{2}}{b^{4}}+\frac{z^{2}}{c^{4}}+\frac{\omega^{2}}{m^{4}}-\frac{y z}{b^{2} c^{2}}-\frac{\omega x}{m^{2} a^{2}} \ldots=0,
$$

where $x$ stands for the more usual $\frac{x}{p_{1}}, y$ for $\frac{y}{p_{2}}, p$ being the perpendicular on a face from a vertex.

The plane of section with the sphere is $\frac{x}{a^{2}}+\ldots=0$, which is the polar plane of $K$, and hence the ellipsoid has a circular section perpendicular to $O K$.

For further convenience, let $x$, which stands for $\frac{x}{p_{1}}$, now stand for $\frac{x}{a^{2} p_{1}}$.

8. An infinite system of tetrahedra with a common symmedian point.

The equation of the sphere is now $\Sigma y z=0$, of the ellipsoid $\Sigma x^{2}-\Sigma y z=0$. The tangent cone from $D$ to the ellipsoid is

$$
x^{2}+y^{2}+z^{2}-2 y z-2 z x-2 x y=0 \text {. }
$$


Where this tangent cone meets the plane of $A B C$, we have an ellipse, and round this ellipse we have the triangle $A B C$, which in turn is inscribed in a circle. We can thus have a poristic series of triangles. Take one of these triangles and call it $P Q R$. We have now a new tetrahedron, $D P Q R$.

Let

$$
\begin{aligned}
& Q R \text { be } l x+m y+n z=0 \\
& R P \text { be } m x+n y+l z=0 \\
& P Q \text { be } n x+l y+m z=0
\end{aligned}
$$

with the condition $\Sigma m n=0$.

The pole of $D Q R$ is given by

$$
\begin{aligned}
x & =\frac{1}{3}(m+n-2 l) \\
y & =\frac{1}{3}(n+l-2 m) \\
z & =\frac{1}{3}(l+m-2 n) \\
\omega & =\frac{1}{3}(l+m+n)
\end{aligned}
$$

and $P$ is given by $x=l, y=m, z=n, \omega=0$.

As the addition of the corresponding coordinates of the pole of $\eta Q R$ and of $P$ give the same result, viz., $\frac{1}{3}(l+m+n)$, the point s. ven by $(1,1,1,1)$, which is $K$, lies on the line joining $P$ to the pole of $D Q R$.

Thus $K$ is the symmedian point of $D P Q R$.

9. A further extension.

In the last paragraph we fixed $D$ and moved round the tangent cone in the plane of $A B C$-getting a new tetrahedron, $D P Q R$.

Start from this and fix $P$ and change $Q R D$ into $L M N$ (say). Proceeding in this manner, we finally get away from $A B C D$ and reach an infinite series of tetrahedra with the same four points, $O, K, H_{1}$, and $H_{2}$.

10. The constant of the system.

Consider $D A B C$ and $D P Q R$. $A B C$ and $P Q R$ are co-Brocardal. Therefore $\quad \frac{a^{2}+b^{2}+c^{2}}{a b c}=\frac{a_{1}^{2}+b_{1}{ }^{2}+c_{1}{ }^{2}}{a_{1} b_{1} c_{1}}$, where $a_{1}=Q R$.

The areas of $A B C$ and $P Q R$ are in the ratio of $a b c$ to $a_{1} b_{1} c_{1}$. The volumes of $D A B C$ and $D P Q R$ are also as $a b c$ to $a_{1} b_{1} c_{1}$, i.e.

$$
\frac{V}{V_{1}}=\frac{a b c}{a_{1} b_{1} c_{1}} \text {. }
$$


But if $a d=k$ and $a_{1} d_{1}=k_{1}$

$$
\begin{aligned}
\frac{k^{2}}{V}= & \frac{k_{1}^{2}}{V_{1}} \\
& (\text { See Salmon's, 4th ed., p. 37). } \\
\therefore \quad \frac{a b c}{k^{2}}= & =\frac{a_{1} b_{1} c_{1}}{k_{1}^{2}} .
\end{aligned}
$$

Consider next$$
\frac{a_{1}^{2}+b_{1}^{2}+c_{1}^{2}+m_{1}^{2}}{a_{1} b_{1} c_{1}}
$$

This expression is equal to

$$
\frac{a^{2}+b^{2}+c^{2}+m^{2}}{a b c} \text {. }
$$

Further, it is symmetrical with respect to the triangles

$$
D Q R, D P Q, P Q R \text {, }
$$

Since $\quad \frac{a^{2}+b^{2}+c^{2}}{a b c}+\frac{a^{2}+\frac{k^{2}}{b^{2}}+\frac{k^{2}}{c^{2}}}{\frac{k^{2} a}{b c}}+\ldots=3 \frac{a^{2}+b^{2}+c^{2}+m^{2}}{a b c}$.

Proceeding in the manner of the last paragraph, we find that this expression, viz.,

$$
\frac{a^{2}+b^{2}+c^{2}+m}{a b c},
$$

is constant for any tetrahedron in the infinite system.

\section{Interpretation of Constant.}

The absolute values of the coordinates of $K$ are

$$
\frac{a^{2} p_{1}}{a^{2}+b^{2}+c^{2}+m^{2}} \text {, etc. }
$$

If these values be substituted for the variables in the 1.h.s. of the equation of sphere ( $v$. Salmon) the result, viz.,

$$
\frac{6 a^{2} b^{2} c^{2}}{\left(a^{2}+b^{2}+c^{2}+m^{2}\right)^{2}}
$$

is equal to the rectangle $A K \cdot A^{\prime} K$.

Mr R. F. DAvis, M.A., of London, has kindly contributed the following note, which shows that if a regular tetrahedron be 
inverted, the origin of inversion is an isodynamic point of the new tetrahedron :-

1. I Let $\alpha \beta \gamma \delta$ be a regular tetrahedron having six equal edges $\beta \gamma, \gamma \alpha, \alpha \beta, \delta \alpha, \delta \beta, \delta \gamma$, and four equal equilateral faces, $\alpha \beta \gamma$, $\delta \beta \gamma, \delta \gamma \alpha, \delta \alpha \beta$.

Take any point $H$ whatsoever within the tetrahedron. Join $H \alpha, H \beta, H \gamma, H \delta$, and produce them respectively to $A, B, C, D$ in such a manner that

$$
H A . H \alpha=H B . H \beta=H C . H \gamma=H D . H \delta=K^{2} \text {, }
$$

so that $A B C D$ is the figure inverse to $\alpha \beta \gamma \delta$ when $H$ is the origin and $K^{2}$ the constant of inversion.

Then $B C: \beta \gamma=H B: H \gamma \quad$ (for $B \beta_{\gamma} C$ are concyclic)

and

$$
=H B \cdot H C: H \gamma \cdot H C=H B . H C / K^{2}
$$

$$
\begin{aligned}
& B C=\left(\beta \gamma / K^{2}\right) H B . H C \\
& C A=(\ldots \ldots \ldots) H C . H A,
\end{aligned}
$$

and so on for all six pairs of corresponding edges.

Notice $B C . D A=(\ldots \ldots)^{2} H A \cdot H B \cdot H C . H D$

$$
=C A \cdot D B=A B, D C \text {, by symmetry. }
$$

2. Conversely, it may be assumed that a tetrahedron $A B C D$ cannot be inverted into a regular tetrahedron $\alpha \beta \gamma \delta$ unless

$$
\begin{gathered}
B C . D A=C A . D B=A B . D C \\
\text { or } a d=b e=c f,
\end{gathered}
$$

where $B C=a, C A=b, A B=c, D A=d, B D=e, D C=f$.

In this case the tetrahedron $A B C D$ is said to be harmonic; and if a suitable centre $H$ of inversion be taken, we have four harmonic tetrahedra within a harmonic tetrahedron, namely,

$\begin{array}{lcc}H A B C & \text { with } & H A \cdot B C=H B \cdot C A=H C \cdot A B \\ H D B C & \Rightarrow & H D \cdot B C=H B \cdot C A=H C \cdot D B \\ H D C A & " & H D \cdot C A=H C \cdot A D=H A \cdot D C \\ H D A B & \Rightarrow & H D \cdot A B=H A \cdot B D=H B \cdot D A\end{array}$

If we put $b c / d=c a / e=a b / f=\mu$, all these relations are included in the one formula

$$
a \cdot H A=b \cdot H B=c . H C=\mu . H D,
$$

which also shows that the position of $H$ is determined as either of the two common points of intersection of four spheres corresponding to the Apollonian circles. 